\title{
Microlocalization and distribution of digestion- resistant aromatic lignin and cellulosic compounds in feeds at cellular and subcellular levels: A novel approach $^{*}$
}

\author{
P. Yu ${ }^{1}$ \\ Department of Animal and Poultry Science, College of Agriculture and Bioresources, \\ University of Saskatchewan \\ 51 Campus Drive, Saskatoon, Canada, S7N $5 A 8$
}

(Received 12 February 2007; revised version 16 November 2007; accepted 21 November 2007)

\begin{abstract}
The objective of this study was to micro-localize the distribution of digestion-resistant aromatic lignin and cellulosic compounds in feeds at cellular and subcellular levels using advanced synchrotron-powered FTIR microspectroscopy (SFTIRM) as a novel approach. The SFTIRM is a newly emerging and nondestructive analytical technique and can reveal molecular chemistry (structural-chemical make-up) of biological samples at highly spatial resolutions $(3-10 \mu \mathrm{m})$ without destruction of the feed internal structures. The exampled feeds used for this pilot study were maize (cv. Pioneer) and barley (cv. Harrington). The results show that with SFTIRM, the images of the aromatic lignin and cellulosic compounds could be generated to be able to show the distribution and intensity across the feeds tissues. The digestion-resistant aromatic lignin compound only presented in the pericarp region and no lignin has been found in seed coat, aleurone layer and endosperm. The cellulosic compounds presented most in the pericarp region, less in the seed coat, aleurone layer, and endosperm. The agglomerative hierarchical cluster analysis (AHCA) and principal component analysis (PCA) showed the distinct differences of the chemical make-up between the two feeds (maize vs barley) and between the different structures (pericarp vs aleurone) within a feed. Even in the same structural regions (such as aleurone layer, and endosperm), the structural make-up were different between the maize and barley. This may explain why digestive behaviours are different between the barley and maize. The implication of this study is that with extremely bright synchrotron light, we can localize and characterize internal feed digestion-resistant compounds in a chemical sense with
\end{abstract}

\footnotetext{
* Supported by Natural Sciences and Engineering Research Council of Canada, Saskatchewan Agricultural Development Fund (ADF), Canadian Light Sources (CLS), University of Saskatchewan, U.S. Department of Energy, Contract DE-AC02-98CH10886

${ }^{1}$ Corresponding author: e-mail: peiqiang.yu@usask.ca
} 
cellular dimensions. With respect to human and animal nutrition, it is possible to relate digestive function and nutrient utilization to the specific chemical make-up of intrinsic structures of feeds. It is believed that with the advanced synchrotron technology, it will make a significant step and an important contribution to feed molecular structural research.

KEY WORDS: aromatic lignin, cellulosic compound, cellular, feed quality, molecular structural make-up, synchrotron, PCA, AHCA

\section{INTRODUCTION}

Lignin and cellulosic compounds in feeds usually play negative roles in determining feed quality, particularly in monogastric animals. Lignin is a highly condensed phenylpropanoid matrix that is relatively resistant to anaerobic fermentation by ruminal microorganisms and to digestive enzymes in intestines. Lignin is also thought to physically encrust cell wall polysaccharides, inhibit their fermentation and usually listed as a digestion-resistant compound in feeds. Lignin content in feeds has been shown to be correlated negatively with feed digestibility (Garleb et al., 1988, 1991). The cellulosic compound in feeds is mainly associated with feed cell wall matrix such as phenolic-carbohydrate complexes, hemicellulose encrustation and cellulose crystallinity and usually listed as another digested-resistant compound. The complex structures of cellulosic compound reduce the susceptibility to digestive enzymes and microbial degradation. A traditional method for analysis of lignin and cellulosic compound is to check their total contents in a feed, but this method is unable to check distribution and intensity of these compounds across feed tissue. Conventional "wet" chemical analysis can determine total chemical composition, but fails to detect feed internal structures due to destruction of feeds during chemical analysis. In other words, the "wet" chemical analysis cannot link structural information to chemical information within intact feeds (Budevska, 2002).

Recently, advanced synchrotron-based Fourier transform infrared microspectroscopy (SFTIRM) has been developed as a non-destructive and non-invasive analytical technique (Wetzel and LeVine, 2000; Wetzel, 2001; Marinkovic et al., 2002; Miller and Dumas, 2006). This technique, taking advantage of bright synchrotron light (million times brighter than sunlight), is capable of exploring the structural make-up within microstructures of a biological tissue without destruction of inherent structures (Raab and Martin, 2001; Yu, 2006). This technique can link chemical information to structural information of biological samples within cellular dimensions. It can provide four kinds information simultaneously: tissue composition, tissue structure, tissue chemistry and tissue environment (Budevska, 2002). However, this novel synchrotron-based technique (SFTIRM) is extremely rarely used for feed research (Yu, 2004).

The objective of this study was to localize digestion-resistant aromatic lignin and cellulosic compounds at different inherent structures of feeds at cellular and 
subcellular levels using the SFTIRM technique. Such information would aid in localization of digestion-resistant compounds in feeds and aid in prediction of feed quality and nutritive value for humans and animals.

\section{MATERIAL AND METHODS}

\section{Feed samples}

Maize (cv. Pinoneer 39P78) at harvest maturity was obtained from Henry Penner (Pox 1028, Morden, MB, R6M 1A9), barley (cv. Harrington, 2000-BI-704) (a tworow, broad and diamond shaped kernel barley bred for malting characteristics with extremely rapid rate of fermentation) was supplied by B. Rossnagel, Crop Development Center (CDC), the University of Saskatchewan (Saskatoon, SK, Canada).

\section{Feed infrared slide and window preparation}

The feed samples were cut into thin cross sections (about $6 \mu \mathrm{m}$ thick) at The Western College of Veterinary Medicine, the University of Saskatchewan. The unstained cross-sections of the tissues were mounted onto Low-e IR microscope slides (Kevley Technologies, Chesterland, $\mathrm{OH}$ ) and $\mathrm{BaF}_{2}$ windows (size: $13 \times 1 \mathrm{~mm}$ disk; part number: 915-3015, Spectral Systems, NY, USA) for synchrotron FTIR microspectroscopy work.

\section{Synchrotron light source and synchrotron FTIR microspectroscopy}

This experiment was performed at the National Synchrotron Light Source in Brookhaven National Laboratory (NSLS-BNL, US Department of Energy, New York). The spectroscopic images were recorded using a Nicolet Magna 860 FTIR (Thermo Nicolet, USA) equipped with a Continuum IR microscope (Spectra Tech, USA), mapping stage controller, $32 \times$ objective and a mercury cadmium telluride (MCT-A) detector. The bench was configured with a synchrotron light beamline with an energy level $800 \mathrm{MeV}$. The spectra were collected in the midinfrared range of $4000-800 \mathrm{~cm}^{-1}$ at a resolution of $4 \mathrm{~cm}^{-1}$ with 64 scans co-added and an aperture setting of about $10 \times 10 \mu \mathrm{m}$. The reasons for the chosen aperture size of $10 \times 10 \mu \mathrm{m}$ were: 1 . the size was within plant cellular and subcellular dimensions; 2. the $10 \mu \mathrm{m} \times 10$ ( $\mu \mathrm{m}$ aperture size was the best for getting good signal to noise ratio spectrum mapping of the tissue. To minimize IR absorption by $\mathrm{CO}_{2}$ and water vapor in ambient air, the optics were purged using dry $\mathrm{N}_{2}$. A background spectroscopic image file was collected from an area free of feed samples. The mapping steps were equal to aperture size. Stage control, data collection 
and processing were performed using OMNIC 6.0 (Thermo-Nicolet, Madison, Wisconsin). Scanned visible images were obtained using a charge-coupled device $(\mathrm{CCD})$ camera linked to the infrared images (objective $\times 32$ ).

Feed spectrum data analysis

The spectral data of the tissues were collected, corrected the background spectrum, and analysed using OMNIC 6.0 (Thermo-Nicolet, Madison, Wisconsin). A baseline correction was applied to generate the final spectra. The data can be displayed as either as a series of spectroscopic images collected at individual wavelength, or as a collection of infrared spectra obtained at each pixel position in the image.

Feed chemical imaging of aromatic lignin and cellulosic compounds

Chemical imaging of lignin indicated by aromatic compound under peaks at about $1510 \mathrm{~cm}^{-1}$ and cellulosic compounds under peaks at about $1246 \mathrm{~cm}^{-1}$ (Figure 1) was determined by OMNIC software 6.0 (Thermo-Nicolet, Madison,

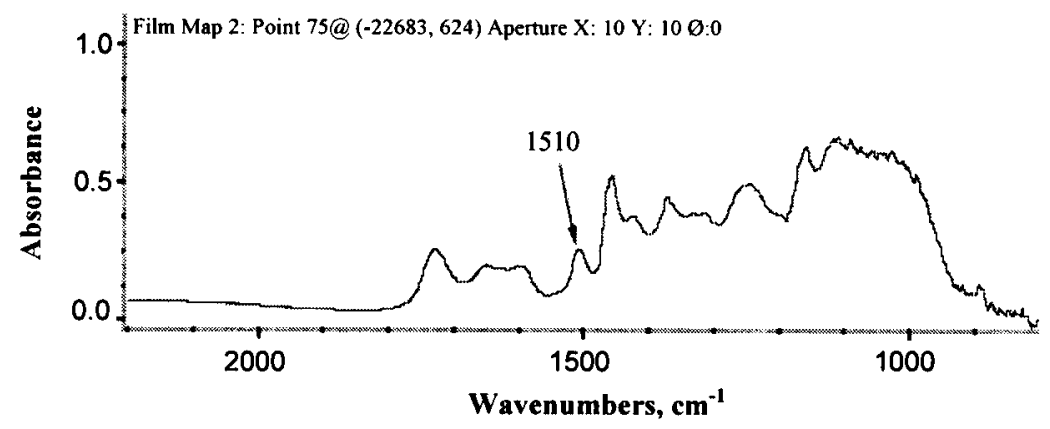

a. Fingerprint band of the undigested aromatic compound (Lignin) about $1510 \mathrm{~cm}^{-1}$

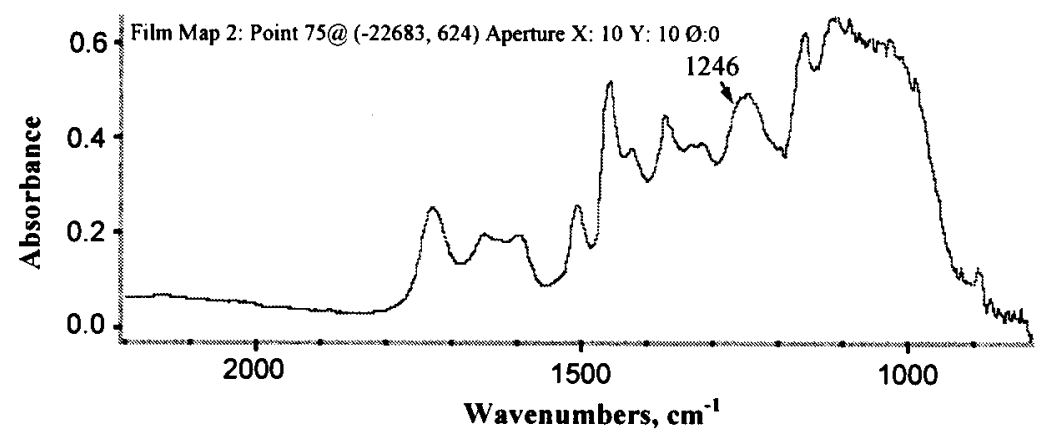

b. Fingerprint band of the uneasily digested structural carbohydrate (cellulosic material) at about $1246 \mathrm{~cm}^{-1}$

Figure 1. Synchrotron FTIR fingerprint bands of the undigested aromatic compound - lignin and the uneasily digested structural carbohydrates-cellulosic material in plant/seed/ feed tissues 
Wisconsin) (Stewart et al., 1995; Himmelsbach et al., 1998; Wetzel and LeVine, 2000; Wetzel, 2001).

\section{Statistical analysis}

Multivariate statistical analyses, agglomerative hierarchical cluster analysis (ANCA) and principal component analysis (PCA), were performed using statistica software 6.0 (StatSoft Inc., Tulsa, OK, USA) to classify the feed inherent structures within a feed and between feeds at cellular and subcellular levels.

\section{RESULTS AND DISCUSSION}

Aromatic lignin and cellulosic compounds in feeds: imaging at cellular and subcellular levels

Lignin and cellulosic compounds in feeds are classified as digestion-resistant compounds in feeds. The study on the distribution and intensity of these compounds in feeds aid in prediction of feed quality or nutritive value in a whole feed or feed fractions for animals. When using synchrotron FTIR microspectroscopy to localize the lignin and cellulosic compounds across feed intrinsic structures, we usually check whether their unique bands appear in the fingerprint regions.

In mid-IR region, the unique fingerprint band of lignin in feeds is at about 1510 $\mathrm{cm}^{-1}$ (Figure 2). This band is considered to be primarily indicative of the aromatic character of the lignin. An aromatic compound gives two major bands at about 1600 and $1500 \mathrm{~cm}^{-1}$, referred to as quadrant and semicircle ring stretch, respectively (Colthup et al., 1990). These are well exemplified in the lignin spectrum that shows bands at about 1600 and $1510 \mathrm{~cm}^{-1}$. The first of these bands is possible interferent with the other bands such as feed protein amide band (about 1650 and $\left.1550 \mathrm{~cm}^{-1}\right)(\mathrm{Yu}, 2005)$. The second of these bands, the peak at about $1510 \mathrm{~cm}^{-1}$, shows no significant inference with any other bands and thus is an excellent diagnostic criterion for aromatics in feeds. Figure 2 is feed chemical images at cellular and subcellular levels $(10 \mu \mathrm{m})$ under area at about $1510 \mathrm{~cm}^{-1}$, showing the spatial lignin distribution and concentration. This image data was taken at the area of the visible image outlined by the rectangle. The $1510 \mathrm{~cm}^{-1}$ band (Colthup et al., 1990; Himmelsbach et al., 1998; Wetzel and LeVine, 2000; Wetzel, 2001) corresponds to the stretch associated with para-substituted benzene rings and can be associated with aromatic species present only in the pericarp region of the both maize and barley. 


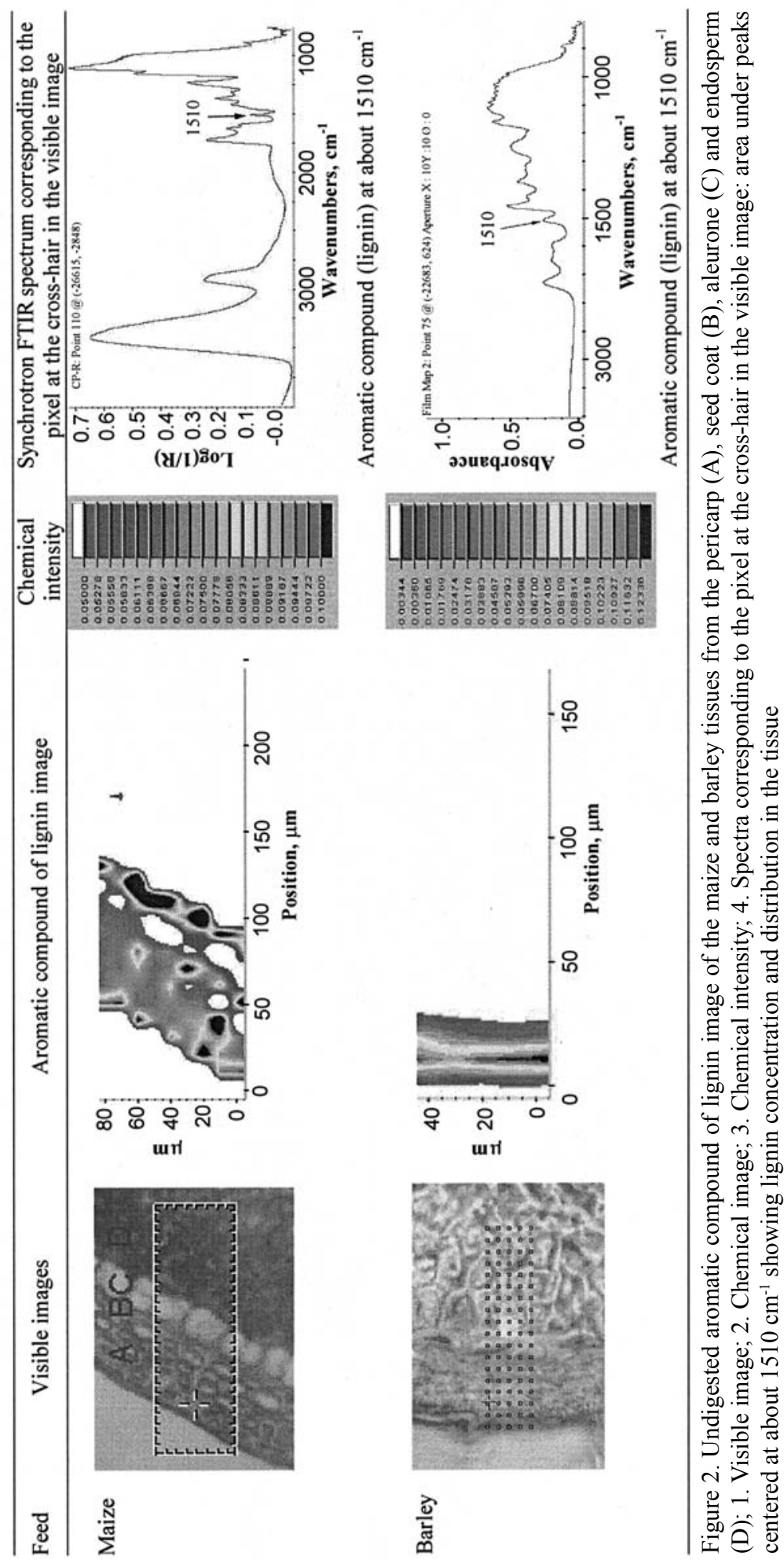


In mid-IR region, the major absorptions from carbohydrates are in the 1200$800 \mathrm{~cm}^{-1}$ region of the spectrum and are attributed to $\mathrm{C}-\mathrm{O}$ stretching vibrations. The unique fingerprint band of cellulosic compound in feeds is at about $1246 \mathrm{~cm}^{-1}$ (Figure 3) (Wetzel and LeVine, 2000; Wetzel, 2001). The cellulosic compound in feeds is mainly associated with feed cell wall matrix such as phenolic-carbohydrate complexes, hemicellulose encrustation and cellulose crystallinity. The complex structures of cellulosic compound reduce the susceptibility to digestive enzymes. Figure 3 is feed chemical image under area at about $1246 \mathrm{~cm}^{-1}$ and shows the spatial cellulosic compound location and concentration, indicating that the pericarp region contains higher cellulosic compound, seed coat, aleurone layer and endosperm contains much less cellulosic compound.

\section{Lignin and cellulosic compounds in feeds: spectral characteristics}

Figures 4 and 5 present the spectra of the pericarp and aleurone regions, showing that similar morphological parts exhibit similar spectral characteristics and similar chemical compounds. All pixels in the pericarp region show spectral peaks at about 1510 and $1246 \mathrm{~cm}^{-1}$ (Figure 4). These indicated that the pericarp contains higher concentration of aromatic lignin $\left(1510 \mathrm{~cm}^{-1}\right)$ and cellulosic compounds $\left(1246 \mathrm{~cm}^{-1}\right)$. All pixels in endosperm region show no spectral peaks at about 1510 and weak spectral peaks at about $1246 \mathrm{~cm}^{-1}$ (Figure 5). The aleurone layer has practically no lignin $\left(1510 \mathrm{~cm}^{-1}\right)$ and much less cellulosic compound $\left(1246 \mathrm{~cm}^{-1}\right)$. Spectral characteristics at different morphological parts (Figures 4 and 5) plus the chemical imaging (Figures 2 and 3 ) can reveal structural features of the feeds and reveal structural chemical make-up.

\section{Discriminate and classify feed structural chemical make-up}

Cluster analysis. The first multivariate analysis, is AHCA, of which function performs an (agglomerative hierarchical) cluster analysis of an IR spectra data set and displays the results of AHCA as dendrograms. First, it calculate distance matrix, which contains information on the similarity of spectra. Then, in hierarchical clustering, the algorithm searches within the distance matrix for the two most similar IR spectra (minimal distance). These spectra are combined into a new object (called a "cluster" or called "hierarchical group"). The spectral distances between all remaining spectra and the new cluster are re-calculated (Cytospec, 2004). It is a technique which clusters IR spectra based on similarity with other spectra. In this study, the Ward's algorithm method was used without any prior parameterization of the spectral data (used original spectral data at fingerprint region 1800 to $800 \mathrm{~cm}^{-1}$ ). This method shows possibility to distinguish the inherent 


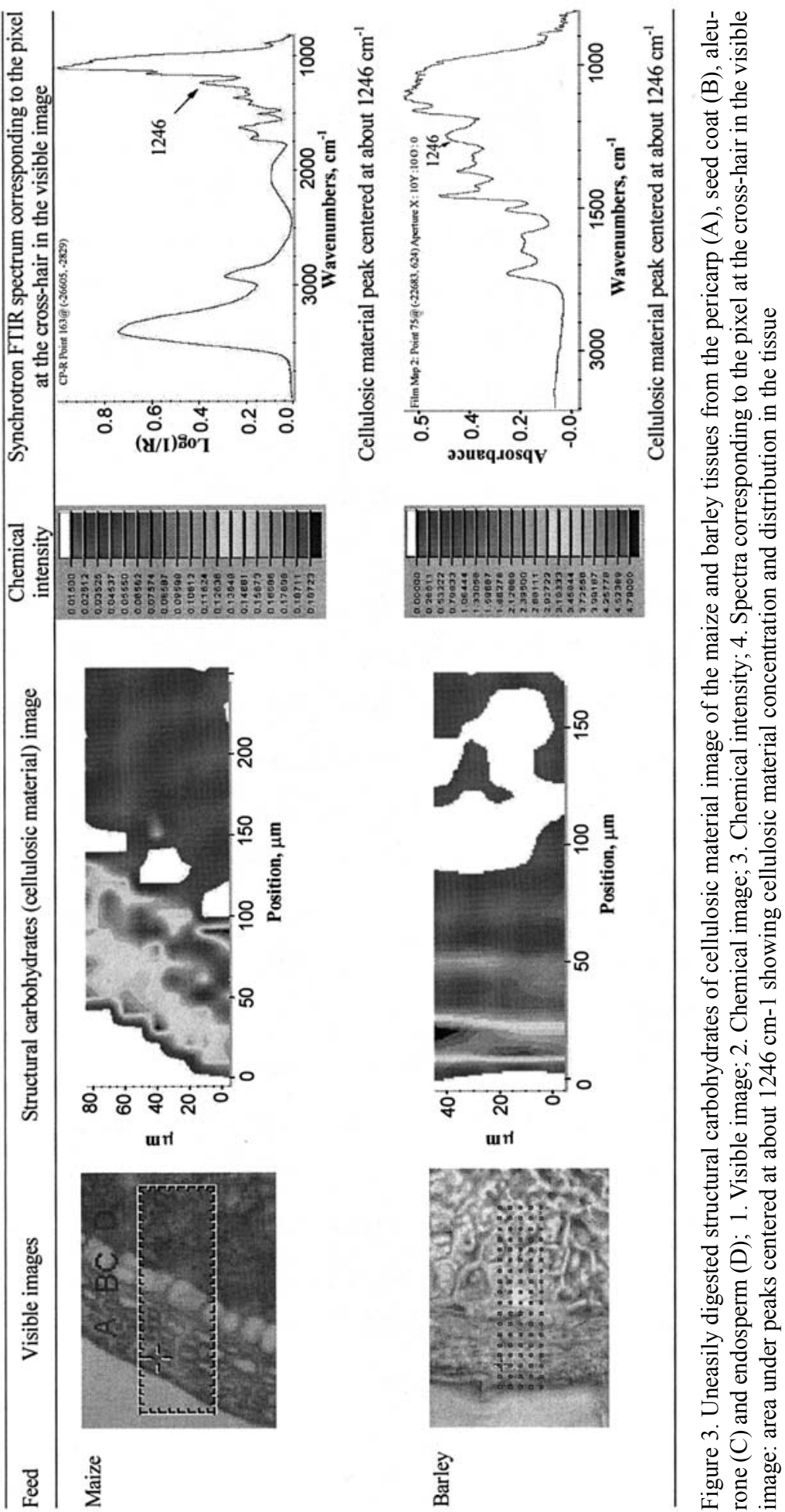




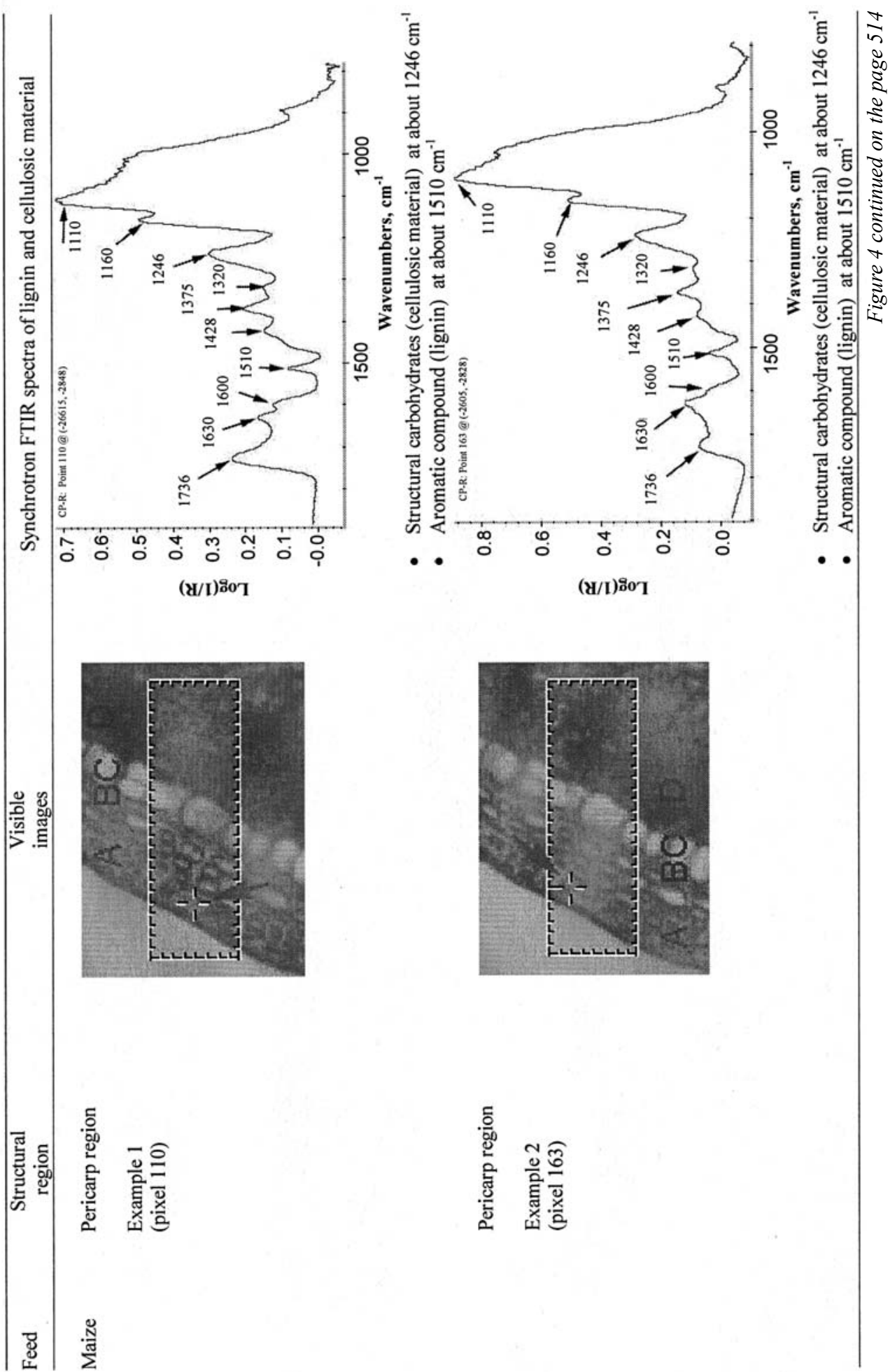




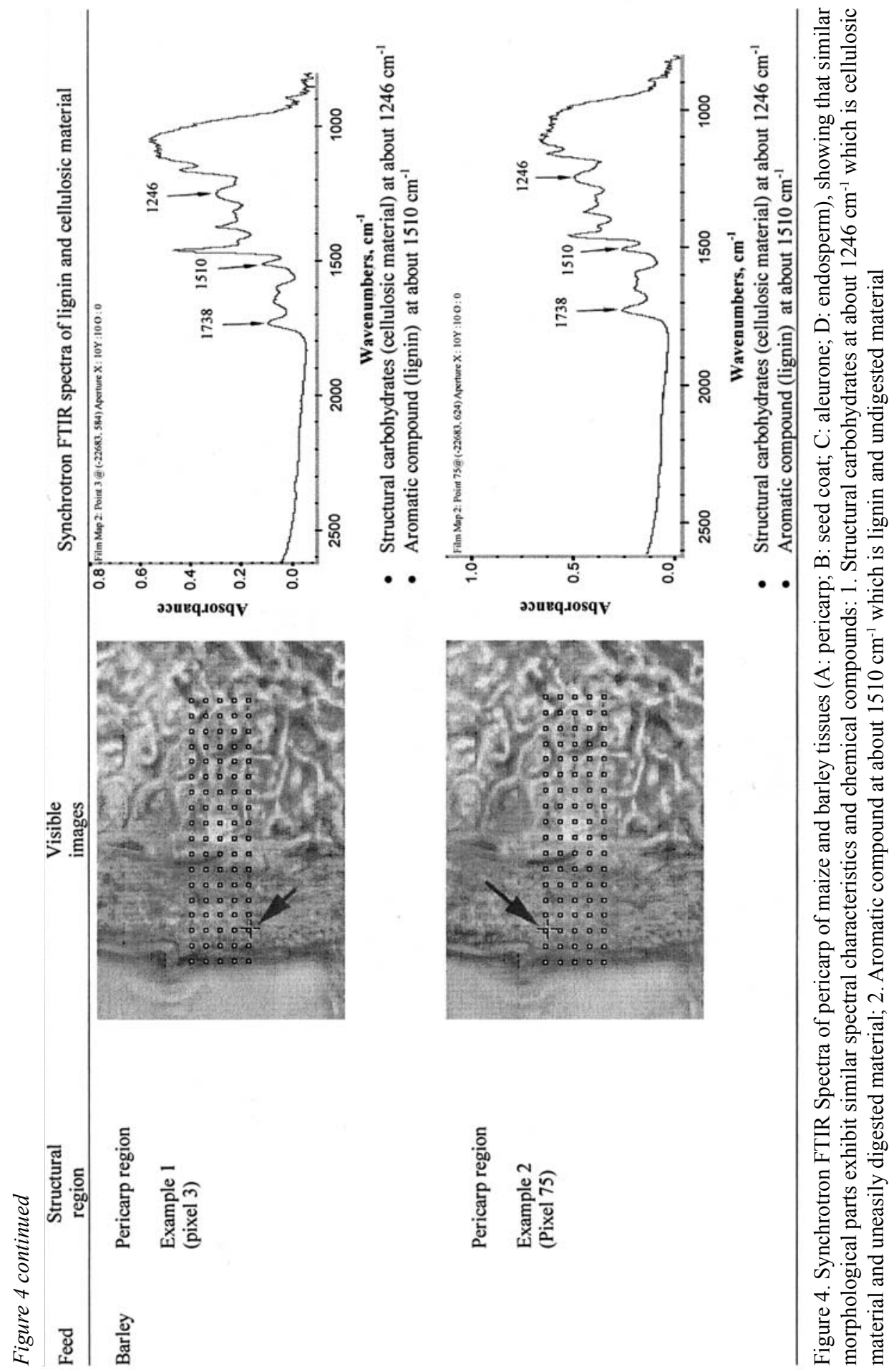




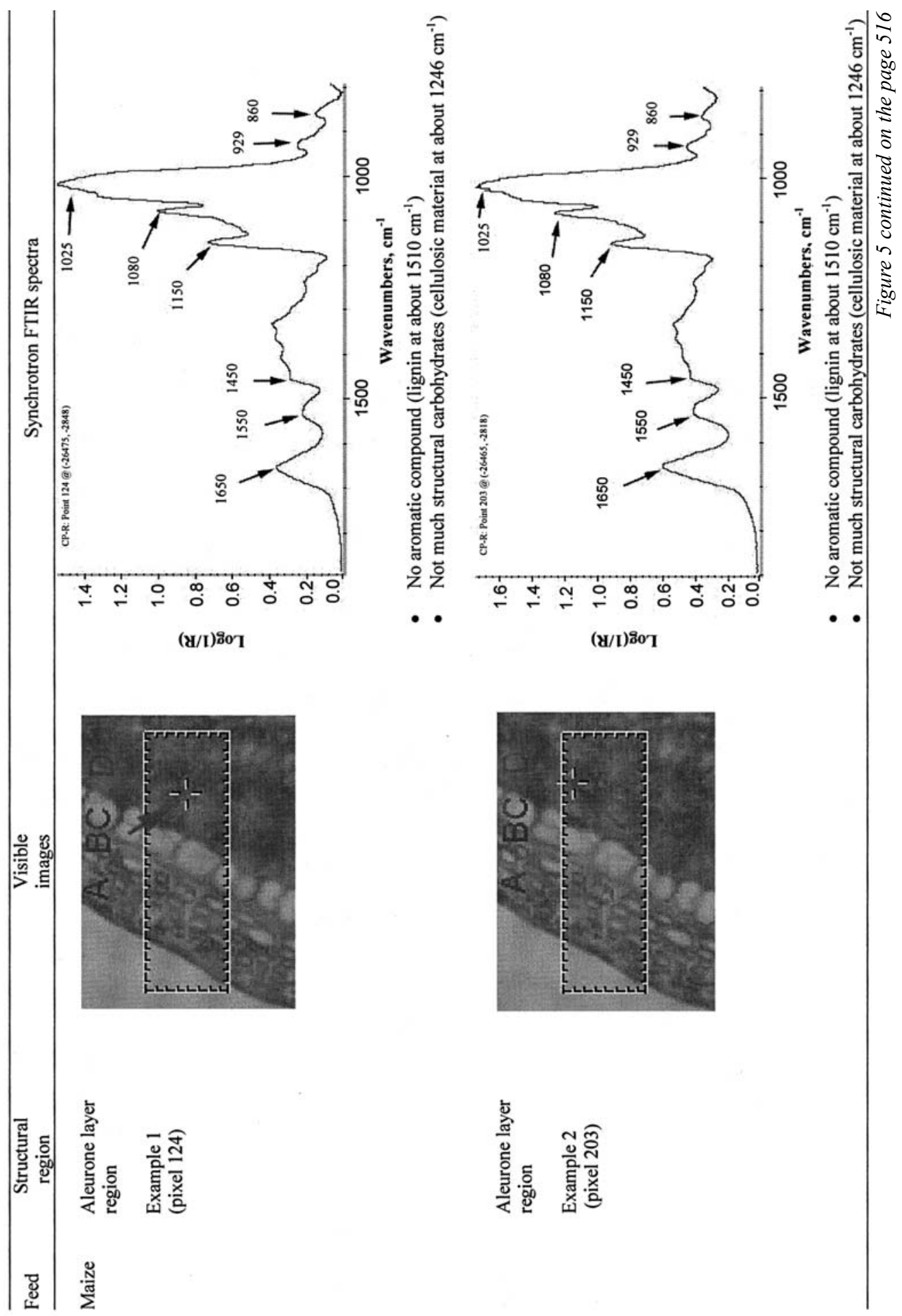




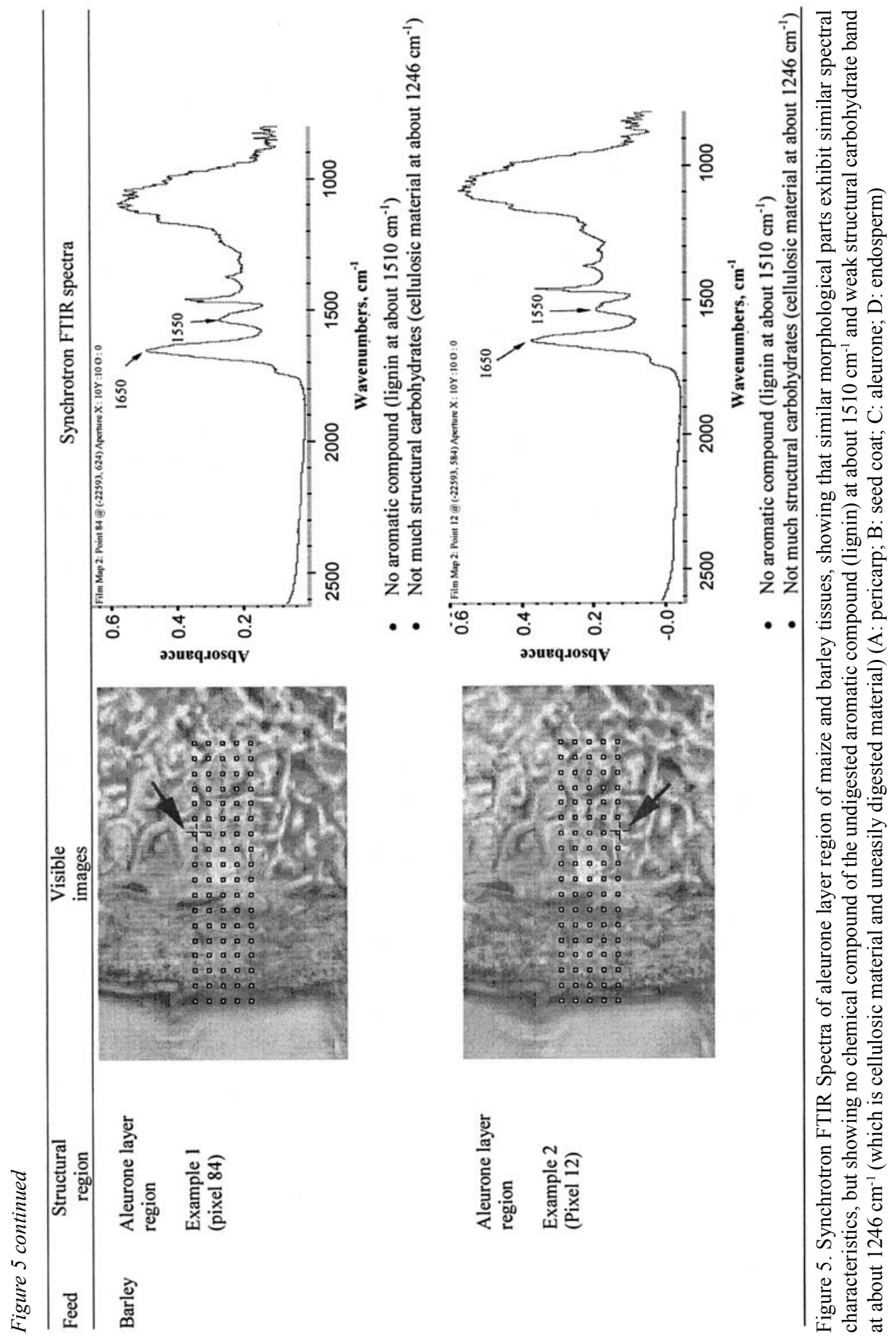


structure difference and chemical make-up between feeds and between different structural layers within a feed.

Differences in the structural chemical make-up between feeds. Figure 6 displays the results in the form of a dendrogram. From this diagram, the two feeds (maize and barley) are fully distinguished in the structural chemical make-up. The CLS clusters of the two feeds are different. This analysis can explains why digestive behaviours are significantly different between the maize and barley mainly due to their different structural chemical make-up.

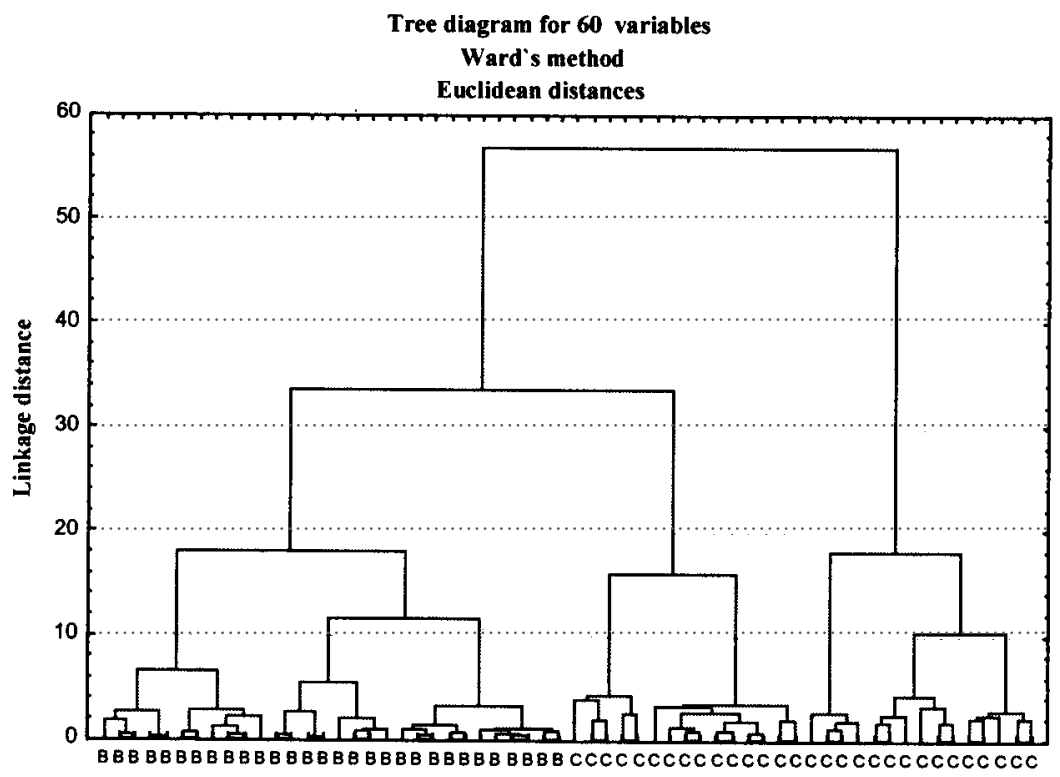

Figure 6. ANCA cluster of feed intrinsic structures showing that the clusters are different between feeds $\left(\mathrm{C}=\right.$ maize, $\mathrm{B}=$ barley) (ANCA analysis: 1 . Spectral region: 1800 to $800 \mathrm{~cm}^{-1} ; 2$. Distance method: Euclidean; 3. Cluster method: Ward's algorithm)

Differences in the structural make-up between different structures within a feed. Figure 7 shows the ANCA clusters within each feed to compare of the spectra from aleurone layer (containing easily digested compounds such as protein and starch) with the spectra from pericarp region (which containing undigested and uneasily digested compounds: lignin and cellulosic compounds). The results clearly show that the clusters are different between different structures (pericarp vs aleurone layer) within a feed. 


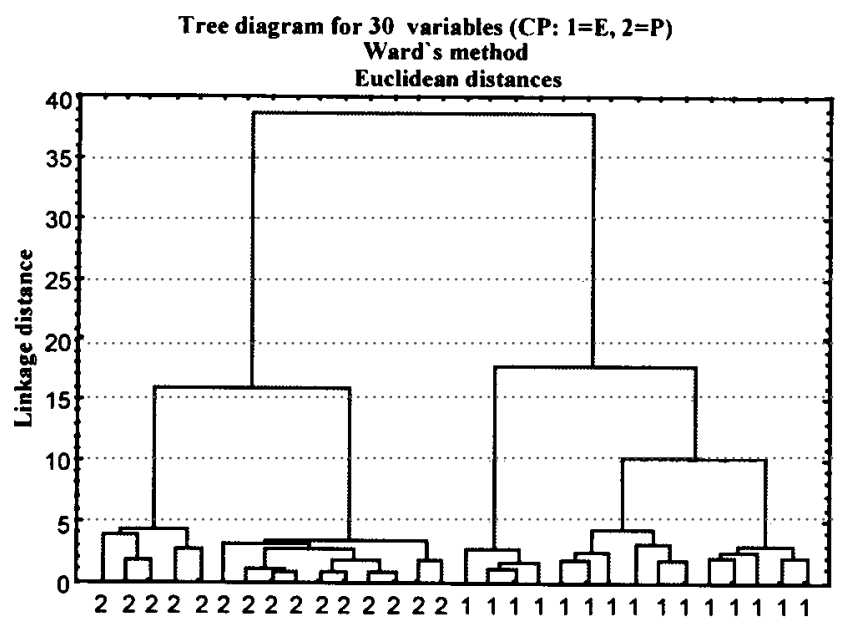

(a) Maze (cv. Pioneer): 1 = Aleurone layer; 2 = Pericarp region

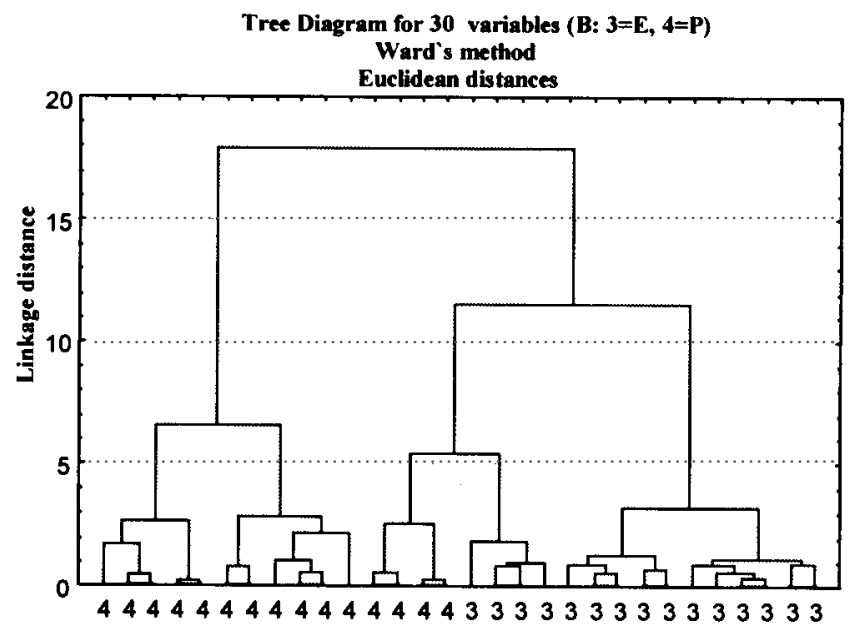

(b) Barley (cv. Harrington): 3 = Aleurone layer; 4 = Pericarp region

Figure 7. ANCA cluster of feed molecular structure obtained from maize (cv. Pioneer) (a) and barley (cv. Harrington) (b): compare of the spectra from aleurone layer (containing easily digested compounds: protein and starch) with the spectra from pericarp region (containing undigested and uneasily digested compounds: lignin and cellulosic compounds), showing that the clusters are different between different structures within a feed (ANCA analysis: 1. Spectral region: about 1800 to 800 $\mathrm{cm}^{-1} ; 2$. Distance method: Euclidean; 3. Cluster method: Ward's algorithm)

Differences in the make-up at the same structure region (or same morphological part) but the different feeds. The AHCA clusters (Figure 8) showing that the clusters in the pericarp are different between the two feeds, and the clusters in the 


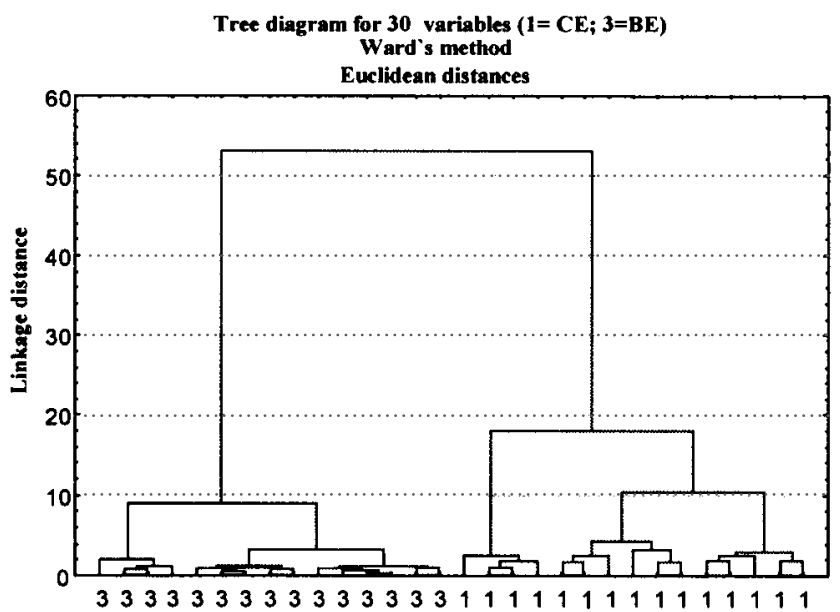

(a) $1=$ Aleurone layer in maize (cv. Pioneer); 3 = Aleurone layer in Barley (cv. Harrington)

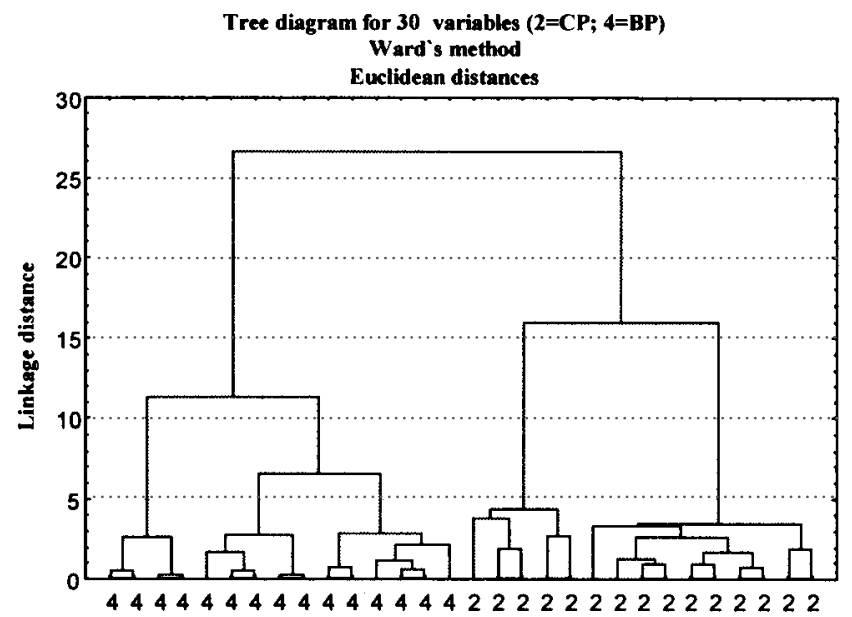

(b) 2 = Pericarp in maize (cv. Pioneer); 4 = Pericarp in Barley (cv. Harrington)

Figure 8. ANCA cluster of feed intrinsic structures showing that the clusters in the pericarp are different between the two feeds (a), and the clusters in the aleurone layer are different between the two feeds (b) (ANCA analysis: 1. Spectral region: 1800 to $800 \mathrm{~cm}^{-1}$; 2. Distance method: Euclidean; 3 . Cluster method: Ward's algorithm)

aleurone layer are also variables called principal components (PCs). The first few PCs will typically different between the two feeds. This indicated that even in the same morphological part, different feeds will have different structural make-up. No publication results have been found that we can compare with. 
Principal component analysis. The second multivariate analyses, is PCA which is a statistical data reduction method. It transforms the original set of variables to a new set of uncorrelated account for $>95 \%$ variance. The purpose of PCA is to derive a small number of independent linear combinations (PCs) of a set of variables that retain as much of the information in the original variables as possible. This analysis allows studying globally the relationships between $p$ quantitative characters (e.g., chemical functional groups, aromatic lignin and cellulosic compopunds) observed on $n$ samples (e.g., FTIR spectra). The basic idea is to extract, in a multiple variable system, one, two or sometimes more PCs that carry maximum information. These components are independent (orthogonal) of each other and the first factor generally represents maximum variance. As factors are extracted, they account for less and less variability and the decision of when to stop basically depends on the point when there is only very little significant variability left, or merely random noise. Thus, reduction of data provides a new coordinate system where axes (eigenvectors) represent the characteristic structure information of the data and the spectra may then be simply described as function of specific properties, and no longer as a function of intensities. The outcome of such an analysis can be presented either as a 2D (two PCs) or 3D (three PCs) scatter plots (Sockalingum et al., 1998). In this feed structure study, PCA was used to identify the main sources of variation in the synchrotron-based FTIR spectra in the fingerprint region $1800-800 \mathrm{~cm}^{-1}$ of the feeds at cellular and subcellular levels

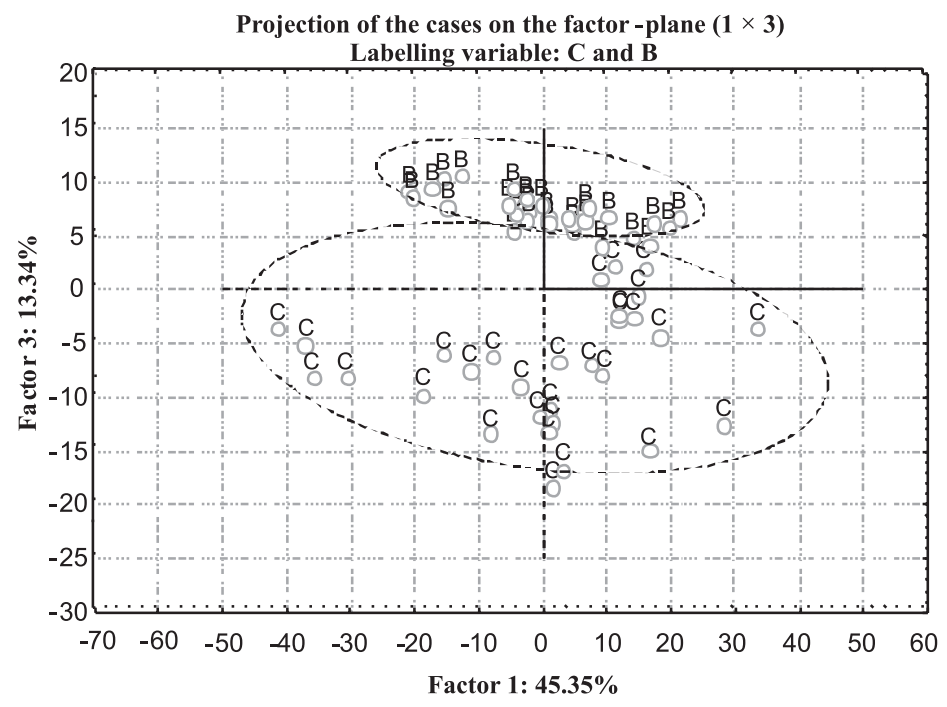

Figure 9. PCA analysis of synchrotron-based feed molecular structure spectrum obtained from maize (cv. Pioneer) and barley (cv. Harrinton) at a cellular level (pixel size $10 \mu \mathrm{m} \times 10 \mu \mathrm{m}$ ): Scatter plot of the $1^{\text {st }}$ principal component (PC1) vs the $3^{\text {rd }}$ principal component (PC3) (PC1 explains $45 \%$ of the variance while PC2 explains $15 \%$ of the variance; PC3 explains $13 \%$ of the variance) 


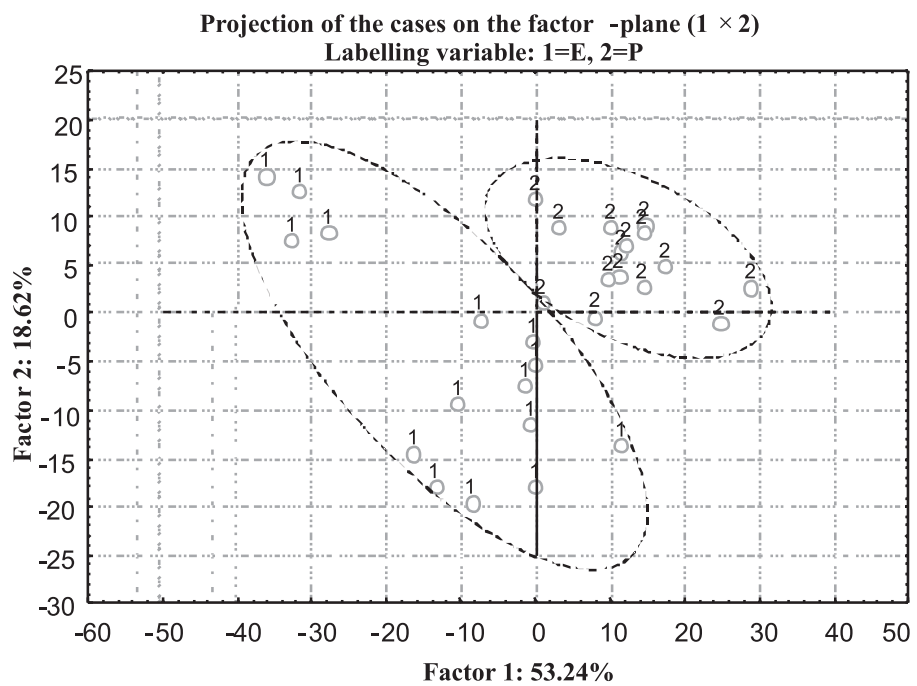

(a) Maize (cv. Pioneer): 1= Aleurone layer; 2 = Pericarp region

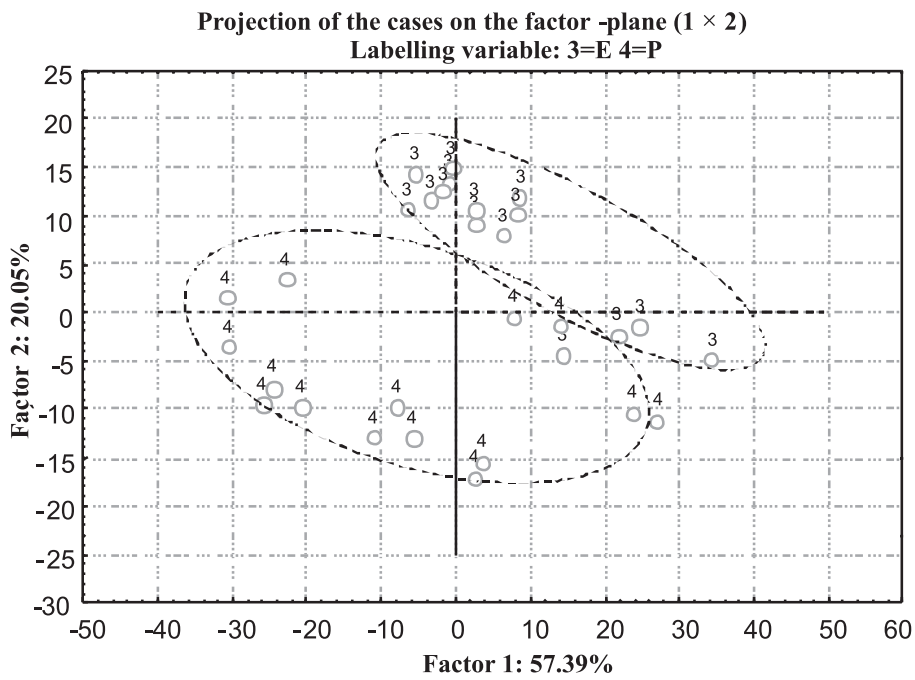

(b) Barley (cv. Harrington): 3= Aleurone layer; 4 = Pericarp region

Figure 10. PCA analysis of synchrotron-based feed molecular structure spectrum obtained from maize (cv. Pioneer) (a) and barley (cv. Harringyon) (b) tissues at a cellular level (pixel size $10 \times 10$ $\mu \mathrm{m}$ ): Scatter plot of the $1^{\text {st }}$ principal component vs the $2^{\text {nd }}$ principal component (for maize: the $1^{\text {st }}$ principal component $(\mathrm{PC} 1)$ explains $53 \%$ of the variance while the $2^{\text {nd }}$ principal component $(\mathrm{PC} 2)$ explains $19 \%$ of the variance; the $3^{\text {rd }}$ principal component (PC3) explains $13 \%$ of the variance; for barley, PC1 explains $57 \%$ of the variance while PC2 explains $20 \%$ of the variance; PC 3 explains $13 \%$ of the variance) 


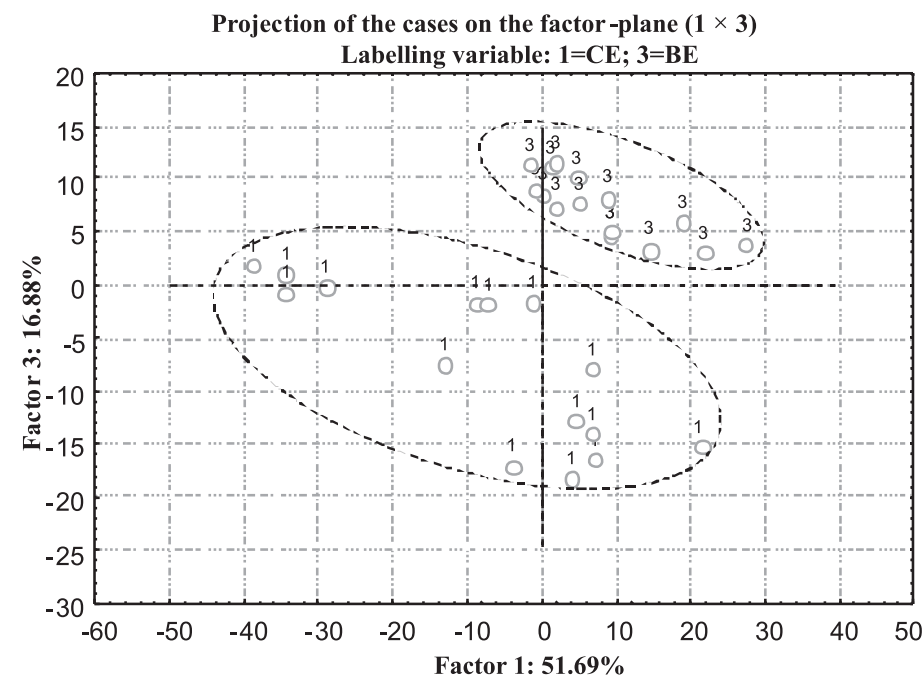

(a) 1=Aleurone layer in maize (cv. Pioneer); 3 = Aleurone layer in barley (cv. Harrington)

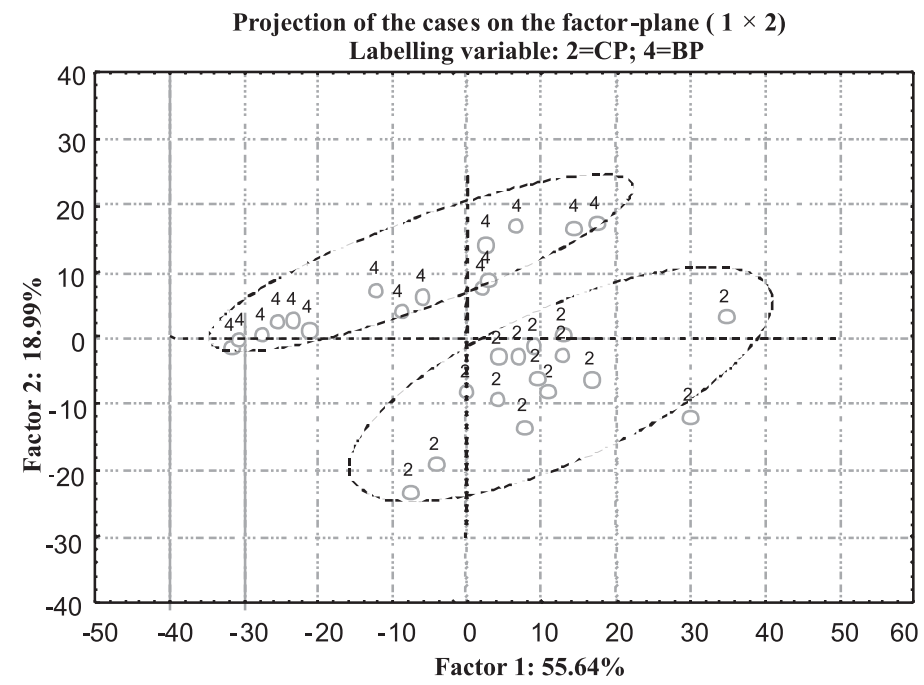

(b) 2 = Pericarp in maize (cv. Pioneer); 4 = Pericarp in barley (cv. Harrington)

Figure 11. PCA analysis of synchrotron-based feed molecular structure spectrum obtained from (a) aleurone layer and (b) pericarp in both maize (cv. Pioneer) and barley (cv. Harrinton) at a cellular level (pixel size $10 \mu \mathrm{m} \times 10 \mu \mathrm{m})$ : Scatter plot of the $1^{\text {st }}$ principal component $(\mathrm{PC} 1)$ vs the $2^{\text {nd }}(\mathrm{PC} 2)$ or $3^{\text {rd }}$ principal component (PC3) (for the aleurone layers in bother maize and barley, PC1 explains $52 \%$ of the variance while PC 2 explains $20 \%$ of the variance; PC 3 explains $17 \%$ of the variance; for the pericarps in bother maize and barley, PC1 explains $56 \%$ of the variance while PC2 explains $19 \%$ of the variance; PC3 explains $11 \%$ of the variance) 
and identify the features that differ between the two feeds (maize vs barley), between different structural regions (or different morphological parts) within the same feed and between the same morphological parts but different feeds. Figure 9 show results from PCA analysis of the spectrum data obtained from the two feeds ( maize vs barley). First three PCs (obtained after data reduction) were plotted (Figure 9), show that the two feeds inherent structures can be grouped in separate ellipses. The first three PCs explain 45, 15, and $13 \%$ of the variation in the structural spectrum data set. Therefore PCA fully distinguished between the maize and barley feed in the structural chemical make-up. The PCA analysis (Figure 10) showed that spectra from the pericarp (containing high undigested lignin and uneasily digested cellulosic compound) and aleurone layer (containing high digested compounds) within each feed can be grouped in separate ellipses. Figure 11 shows that even in the same morphological part (or the same structural region), the PCA analysis showed that spectra from the pericarp in the maize and the pericarp in barley and the spectera from the aleurone layer in the maize and the aleurone in the barley are grouped in separate ellipses. These results clearly indicate that the structural chemical make-up between the feeds, even in the same region, are fully distinguished. There is no publication that has been found to use PCA analysis for feed quality research. No comparison can be made.

\section{Implications}

The implications from this study are that the spectroscopic profile differences in feed structural chemical make-up between feeds and feed fractions could be determined by synchrotron-based FTIR microspectroscopy. With respect to human and animal nutrition, it is possible to relate digestive function and nutrient utilization to the specific chemical make-up of intrinsic structures of feeds. With an extreme bright Synchrotron light (S-FTIR), now we can "see" inside feeds (aromatic lignin and cellulosic compounds) in a chemical senses at cellular or subcellular levels $(5-30 \mu \mathrm{m})$. We can chemically define feed intrinsic structures and analyse individual feed parts within intact feed tissues. We can compare feeds (species/varieties/treatments/processing effects) according to spectral characteristics, spatial distribution and chemical intensity of biological compounds within cellular and subcellular dimensions. Further more study is needed to understand and further quantify the relationship between feed structural chemical make-up and nutritive value, digestive or fermentation behaviour in animals. It is believed that with the advanced synchrotron technology, it will make a significant step and an important contribution to feed molecular structural-chemical research. 


\section{CONCLUSIONS}

With synchrotron FTIR microspectroscopy, the digestion-resistant aromatic lignin and cellulosic compounds across different feed structural regions at cellular and subcelluar levels can be imaged, localized and rapidly characterized. Lignin and cellulosic compounds can be linked to structural information in the feeds without destruction of intrinsic structures of feeds. Both AHCA and PCA analyses can distinguish the differences of the structural chemical make-up between the two feeds ( maize vs barley), between different inherent structural regions within a feed, between the two feeds but in the same structural regions. The implication of this study is that with extremely bright synchrotron light, we can characterize and compare feeds (species/varieties/ treatments/processing effects) according to spectral characteristics and relate this information to feed quality. Such information on the structural chemical make-up of feeds may also be used for feed breeding program for selecting superior variety of feed for targeted food and feed purposes and for prediction of feed quality and nutritive value for humans and animals.

\section{ACKNOWLEDGMENTS}

The synchrotron-based feed research program has been financially supported from Natural Sciences and Engineering Research Council of Canada (NSERC, Individual Discovery Grant), Saskatchewan Agricultural Development Fund (ADF) and Canadian Light Sources (CLS) and University of Saskatchewan' synchrotron research travel fund. The National Synchrotron Light Source in Brookhaven National Laboratory (NSLS-BNL, New York, USA) is supported by the U.S. Department of Energy contract DE-AC02-98CH10886.

The author is grateful to John McKinnon, Colleen Christensen and David Christensen (University of Saskatchewan) for strongly support, Nebojsa Marinkovic, Lisa Miller, Wang Qi, Alexander Ignatov and Jennifer Bohon at NSLS-BNL (New York) for helpful data collection, and Brian Rossnagel (CDC) and Vern Racz (PFRC) for supplying the samples. 


\section{REFERENCES}

Budevska B.O., 2002. Applications of vibrational spectroscopy in life, pharmaceutical and natural sciences. In: J.M. Chalmers, P.R. Griffiths (Editors). Handbook of Vibrational Spectroscopy, Vol. 5. John Wiley and Sons, Inc., New York, NY, pp. 3720-3732

Colthup N.B., Daly L.H., Wiberley S.E., 1990. Introduction to Infrared and Raman Spectroscopy. 3rd Edition. Academic Press, Boston, pp. 547

Cytospec, 2004. Software for Infrared Spectral Imaging. V. 1.1.01

Garleb K.A., Bourquin L.D., Hsu J.T., Wagner G.W., Schmidt S.J., Fahey Jr. G.C., 1991. Isolation and chemical analysis of nonfermented fiber fractions of oat hulls and cottonseed hulls. J. Anim. Sci. 69, 1255-1271

Garleb K.A., Fahey Jr. G.C., Lewis S.M., 1988. Chemical composition and digestibility of fiber fractions of certain by-product feedstuffs fed to ruminants. J. Anim. Sci. 66, 2650- 2662

Himmelsbach D.S., Khalili S., Akin D.E., 1998. FT-IR microspectroscopic imaging of flax (Linum usitatissimum L.) stems. Cell. Mol. Biol. 44, 99-108

Marinkovic N.S., Huang R., Bromberg P., Sullivan M., Toomey J., Mille L.M., Sperber E., Moshe S., Jones K.W., Chouparova E., Lappi S., Franzen S., Chance M.R., 2002. Center for Synchrotron Biosciences' U2B beamline: an international resource for biological infrared spectroscopy. J. Synchrotron Radiat. 9, 189-197

Miller L.M., Dumas P., 2006. Chemical imaging of biological tissue with synchrotron infrared light. Biochim. Biophys. Acta (in press)

Raab T.K., Martin M.C., 2001. Visualizing rhizosphere chemistry of legumes with mid-infrared synchrotron radiation. Planta 213, 881-887

Sockalingum G.D., Bouhedja W., Pina P., Allouch P., Bloy C., Manfait M., 1998. FT-IT spectroscopy as an emerging method for rapid characterization of microorganisms. Cell. Mol. Biol. 44, 261-269

Stewart D., McDougall G.J., Baty A., 1995. Fourier transform infrared microspectroscopy of anatomically different cells of flax (Linum usitatissimum) stems during development. J. Agr. Food Chem. 43, 1853-1858

Wetzel D.L., 2001. When molecular Causes of wheat quality are known, molecular methods will supercede traditional methods. Proceedings of International Wheat Quality Conference II, Manhattan, Kansas (USA), pp. 1-20

Wetzel D.L., LeVine S.M., 2000. Biological applications of infrared microscpectroscopy. In: H.U. Gremlich, B. Yan (Editors). Infrared and Raman Spectroscopy of Biological Materials. Marcel Dekkar, New York, pp. 101-142

Yu P., 2004. Application of advanced synchrotron-based Fourier transform infrared microspectroscopy (SR-FTIR) to animal nutrition and feed science: a novel approach. Brit. J. Nutr. 92, 869885

Yu P., 2005. Application of cluster analysis (CLA) in feed chemical imaging to accurately reveal structural-chemical features of feeds within cellular dimension. J. Agr. Food Chem. 53, 28722880

Yu P., 2006. Synchrotron IR microspectroscopy for protein structure analysis: potential and questions. A review. Spectroscopy 20, 229-251 (Invited review article) 